\title{
Polymerization of Unsaturated Sugars I. Radical Copolymerization of D-Glucal Derivatives and Maleic Anhydride
}

\author{
Yoshiyuki Koyama, Mitsuo KaWATA, and Keisuke KuRITA \\ Department of Industrial Chemistry, Faculty of Engineering, \\ Seikei University, Musashino-shi, Tokyo 180, Japan
}

(Received November 13, 1986)

\begin{abstract}
Tri- $O$-acetyl-, tri- $O$-benzyl-, tri- $O$-methyl-D-glucal were copolymerized with maleic anhydride (MAnh) to afford novel vinyl copolymers containing sugar residues in the main chain. Tri-O-benzyl-D-glucal (TBG) showed higher copolymerization reactivity with MAnh than the other glucal derivatives. The copolymerization of TBG and MAnh was carried out at various monomer feed ratios to give the copolymers always rich in MAnh units. Both the complex and penultimate models could explain the compositional data, but the analysis of the copolymerization rate suggested the participation of the CT complex in the propagation.

KEY WORDS Radical Copolymerization / Unsaturated Sugar / Glucal / Maleic Anhydride / Charge-Transfer Complex / Penultimate Model / Complex Model /
\end{abstract}

Since Fischer and Zach first reported the preparation of a D-glucal (1,2-unsaturated-Dglucose) derivative in $1913,{ }^{1}$ many kinds of unsaturated sugars have been synthesized extensively. Glycals are cyclic vinyl ethers having a double bond between C-1 and C-2 and useful in synthetic carbohydrate chemistry for their capability of a variety of addition reactions. However, no attempt has been made to discuss the possibility of the polymerization of glycals.

We have synthesized polymers having sugar units in the main chain by addition and condensation polymerization of mono- $^{2-4}$ and disaccharides. $^{5-7}$ These polymers are halfnatural and half-synthetic and have characteristics of both polysaccharides and synthetic polymers. Polymerization of unsaturated sugars seemed another possible way to synthesize such carbohydrate-containing polymers. Glucals were thus attempted to copolymerize with maleic anhydride (MAnh), since some cyclic vinyl ethers such as $p$-dioxene, ${ }^{8}$ 2,3-dihydropyran, ${ }^{9,10}$ and 2,3-dihydrofuran ${ }^{11}$ were reported to copolymerize with MAnh through charge-transfer complex polymerization to form alternating copolymers.

In this study, tri- $O$-acetyl-, tri- $O$-benzyl-, and tri- $O$-methyl-D-glucal were copolymerized with MAnh under various conditions to evaluate their reactivity and substituent effects in radical copolymerization. The mechanism of the copolymerization is also discussed.

\section{EXPERIMENTAL}

\section{Materials}

Commercial 3,4,6-tri- $O$-acetyl-D-glucal (TAG) was purified by recrystallization from benzene and $n$-hexane. 2,3-Dihydropyran (DHP) was purified by distillation. MAnh and 2,2'-azobisisobutyronitrile (AIBN) were recrystallized from dry benzene and methanol, respectively. Solvents were purified by conventional methods. 


\section{3,4,6-Tri-O-benzyl-D-glucal (TBG)}

To a solution of $25 \mathrm{~g}$ of TAG in $170 \mathrm{ml}$ of dry methanol was added $30 \mathrm{ml}$ of methanol containing $3.5 \mathrm{~g}$ of sodium methoxide. After standing overnight at room temp., the solution was neutralized with an $\mathrm{H}^{+}$-type cation exchange resin and concentrated. The residual oil was dissolved in $360 \mathrm{ml}$ of dimethylformamide, and treated with $11.3 \mathrm{~g}$ of an oil suspension of sodium hydride $(55 \%)$ at room temp. for $2 \mathrm{~h}$. Benzyl chloride $(31.8 \mathrm{ml})$ was added to the mixture slowly at $0^{\circ} \mathrm{C}$. The reaction mixture was left at room temp. overnight and diluted with $500 \mathrm{ml}$ of benzene. It was extracted with deionized water several times, and the benzene solution was concentrated to a syrup, which was crystallized from ethanol. Two recrystallizations gave $16.3 \mathrm{~g}$ of colorless needles $(42.6 \%), \mathrm{mp} 59.7-$ $61.0^{\circ} \mathrm{C}$ (lit. ${ }^{12} \mathrm{mp} 55^{\circ} \mathrm{C}$ ).

\section{3,4,6-Tri-O-methyl-D-glucal (TMG)}

TAG $(25 \mathrm{~g})$ was treated with sodium methoxide in methanol as described above, and the resulting syrup was dissolved in $375 \mathrm{ml}$ of dimethylformamide. An oil suspension of sodium hydride $(55 \%), 22 \mathrm{~g}$, was added to the solution, and the mixture was left at room temp. for $2 \mathrm{~h}$. Methyl iodide $(38.5 \mathrm{ml})$ was then added to the mixture at $0^{\circ} \mathrm{C}$. After standing at room temp. for $6 \mathrm{~h}$, the reaction mixture was diluted with $500 \mathrm{ml}$ of benzene, washed with water, and concentrated to a syrup. It was distilled under reduced pressure. Yield $9.84 \mathrm{~g}$ $\left(56.9 \%\right.$ ), bp $71^{\circ} \mathrm{C} / 3 \mathrm{mmHg}$ (lit. ${ }^{13}$ bp $120^{\circ} \mathrm{C} /$ $20 \mathrm{mmHg}$ ).

\section{Determination of Association Constants for}

Charge-Transfer Complex Formation

The association constant $K$ was determined by ${ }^{13} \mathrm{C}$ NMR spectroscopy as reported by Foster and Fyfe. ${ }^{14}$ The NMR spectra were measured in $\mathrm{CDCl}_{3}$ at $25^{\circ} \mathrm{C}$ at the following monomer concentrations: $[\mathrm{TAG}]=0.030$ $\mathrm{moll}^{-1},[\mathrm{TBG}]=0.030 \mathrm{moll}^{-1},[\mathrm{TMG}]=0.10$ moll ${ }^{-1},[\mathrm{DHP}]=0.026 \mathrm{moll}^{-1}$, and $[\mathrm{MAnh}]=$
$2-4 \mathrm{moll}^{-1}$.

\section{Copolymerization}

Given amounts of monomers, AIBN, and solvent were charged in a glass ampul, and the solution was degassed by the freeze-thaw method three times. The ampul was sealed under vacuum and placed in a thermostat. After polymerization, the contents of the ampul were diluted with acetone and poured into a mixture of hexane and ether $(1: 1)$ to precipitate the polymer. It was purified by three reprecipitations with acetone and hexane-ether $(1: 1)$ and dried under vacuum.

\section{Measurements}

${ }^{1} \mathrm{H}$ and ${ }^{13} \mathrm{C}$ NMR spectra were measured at 270 and $68 \mathrm{MHz}$, respectively, with a JEOL JNM-GX270 spectrometer using tetramethylsilane as the internal reference. Gel permeation chromatography was run in tetrahydrofuran with a JASCO BIP-I liquid chromatograph (columns: Shodex GPC AD-803/s and AD$805 / \mathrm{s})$.

\section{RESULTS AND DISCUSSION}

\section{Charge-Transfer Complex Formation}

The spectra due to the charge-transfer between MAnh and glucal derivatives (TAG, TBG, and TMG) or DHP were measured in benzene at room temperature, and a broad absorption corresponding to charge-transfer transition was observed in a range 380$450 \mathrm{~nm}$ except in the case with TAG-MAnh. The absorptions of the complexes were, however, very weak and overlapped with those of the electron donor molecules. This made difficult the determination of the equilibrium constant $K$ for complex formation. The constant $K$ was thus determined according to the method used by Foster and Fyfe ${ }^{14}$ where the chemical shifts of the donor molecules in the ${ }^{13} \mathrm{C}$ NMR spectra were measured with different MAnh concentrations.

When $[A] \gg[D]$, 


$$
\frac{\Delta}{[\mathrm{A}]_{\mathrm{D}}}=-\Delta \cdot K+\Delta_{0} \cdot K
$$

where $\Delta=\delta_{\text {obs }}^{\mathrm{D}}-\delta_{0}^{\mathrm{D}}, \Delta_{0}=\delta_{\mathrm{AD}}^{\mathrm{D}}-\delta_{0}^{\mathrm{D}},[\mathrm{A}]$ and [D] are the concentrations of acceptor and donor, respectively, $\delta_{\text {obs }}^{\mathrm{D}}$ is the observed shift of a donor carbon in the complexing medium, $\delta_{0}^{\mathrm{D}}$ is that of the donor carbon in the uncomplexed state, and $\delta_{\mathrm{AD}}^{\mathrm{D}}$ is that of the donor

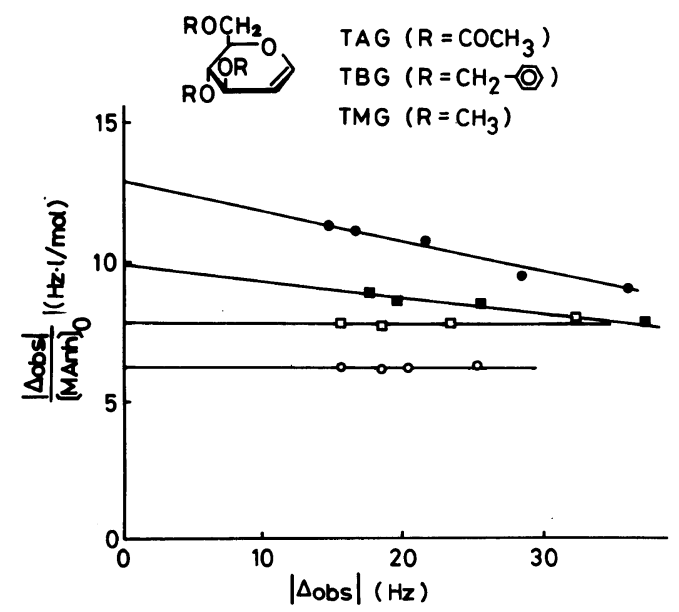

Figure 1. Plots of $\Delta /[\mathrm{MAnh}]$ against $\Delta$ for the mixtures of maleic anhydride with tri- $O$-acetyl-D-glucal $(O)$, tri$O$-benzyl-D-glucal ( $\bigcirc$ ), tri- $O$-methyl-D-glucal $(\square)$, and 2,3-dihydropyran ( $\square$ ) in $\mathrm{CDCl}_{3}$ at $25^{\circ} \mathrm{C}$. carbon in the pure complex.

The ${ }^{13} \mathrm{C}$ NMR spectra were measured on the solutions of donor and acceptor molecules in $\mathrm{CDCl}_{3}$ at $25^{\circ} \mathrm{C}$, and the chemical shift of the $\alpha$ vinyl carbon of the donor monomer was applied here. A plot of $\Delta /[\mathrm{A}]$ against $\Delta$ was linear as shown in Figure 1, indicating the $1: 1$ complex formation. The equilibrium constants $K$ could be obtained directly from the slopes of the lines, and the values are listed in Table I. As shown in the table, the $K$ value for the TBG-MAnh system is relatively high $(0.11$ $1 \mathrm{~mol}^{-1}$ ), but the value for TAG-MAnh and TMG-MAnh is zero, though TAG and TMG have similar ring-structures to TBG. The facts that the $\Delta$ values for TAG-MAnh and TMG-MAnh increased with [A] and that

Table I. Equilibrium constants $K$ for the chargetransfer complex formation from D-glucal derivatives and dihydropyran with maleic anhydride in $\mathrm{CDCl}_{3}$ at $25^{\circ} \mathrm{C}$

\begin{tabular}{cc}
\hline Donor & $\begin{array}{c}K \\
\text { in } 1 \mathrm{~mol}^{-1}\end{array}$ \\
\hline TAG & 0.00 \\
TBG & 0.11 \\
TMG & 0.00 \\
DHP & 0.053 \\
\hline
\end{tabular}

Table II. Copolymerization of D-glucal derivatives and dihydropyran $\left(\mathrm{M}_{2}\right)$ with maleic anhydride $\left(\mathrm{M}_{1}\right)^{\mathrm{a}}$

\begin{tabular}{|c|c|c|c|c|c|c|}
\hline No. & $\mathrm{M}_{2} / \mathrm{mmol}$ & $\begin{array}{c}\text { AIBN } \\
\text { in } \times 10^{-2} \mathrm{mmol}\end{array}$ & Solvent $/ \mathrm{ml}$ & $\begin{array}{l}\text { Time } \\
\text { in } h\end{array}$ & $\begin{array}{l}\text { Conversion } \\
\text { in } w t \%\end{array}$ & $\bar{M}_{n}^{\mathrm{b}}$ \\
\hline 1 & TAG (2) & 4 & None & 48 & 13.6 & 1320 \\
\hline 2 & TAG (2) & 4 & Benzene $(0.8)$ & 24 & 6.0 & 850 \\
\hline 3 & TAG (2) & 8 & Benzene (0.8) & 48 & 16.2 & 1070 \\
\hline 4 & TAG (2) & 4 & $\mathrm{CH}_{2} \mathrm{Cl}_{2}(0.8)$ & 24 & 6.3 & 750 \\
\hline 5 & TBG (1) & 4 & None & 24 & 40.0 & 1740 \\
\hline 6 & TBG (1) & 2 & Benzene (0.5) & 48 & 35.2 & 1860 \\
\hline 7 & TBG (1) & 4 & Benzene (0.5) & 48 & 44.0 & 1630 \\
\hline 8 & TBG (1) & 4 & $\mathrm{CH}_{2} \mathrm{Cl}_{2}(0.5)$ & 48 & 39.8 & 1830 \\
\hline 9 & TBG (1) & 4 & Acetone (0.5) & 48 & 29.8 & 2340 \\
\hline 10 & TMG (2) & 8 & None & 20 & 14.8 & 1470 \\
\hline 11 & TMG (0.8) & 4 & Benzene (0.5) & 48 & 12.0 & 1370 \\
\hline 12 & DHP (2) & 8 & None & 20 & 36.1 & 3810 \\
\hline 13 & DHP (2) & 8 & Acetone $(0.8)$ & 20 & 42.6 & 2190 \\
\hline
\end{tabular}

a $\left[\mathrm{M}_{1}\right]=\left[\mathrm{M}_{2}\right]$ at $60^{\circ} \mathrm{C}$.

b Determined by GPC using polystyrene as standard samples. 
Table III. Copolymerization of tri- $O$-benzyl-D-glucal $\left(\mathrm{M}_{2}\right)$ with maleic anhydride $\left(\mathrm{M}_{1}\right)^{\mathrm{a}}$

\begin{tabular}{|c|c|c|c|c|c|}
\hline \multirow{2}{*}{$\begin{array}{c}\mathrm{M}_{1} \\
\text { in } \mathrm{mol} \%\end{array}$} & \multirow{2}{*}{$\begin{array}{c}\mathbf{M}_{2} \\
\text { in } \mathrm{mol}^{\circ} \%\end{array}$} & \multirow{2}{*}{$\begin{array}{c}\text { Conversion } \\
\text { in } w t \%\end{array}$} & \multicolumn{2}{|c|}{ Copolymer composition } & \multirow[b]{2}{*}{$\overline{\mathrm{DPn}}$} \\
\hline & & & $\begin{array}{c}\mathrm{M}_{1} \\
\text { in } \mathrm{mol} \%\end{array}$ & $\begin{array}{c}\mathbf{M}_{2} \\
\text { in } \mathrm{mol} \%\end{array}$ & \\
\hline 75.0 & 25.0 & 31.9 & 66.3 & 33.7 & 9.5 \\
\hline 63.5 & 37.5 & 27.0 & 62.6 & 37.4 & 9.6 \\
\hline 50.0 & 50.0 & 22.5 & 59.5 & 40.5 & 8.7 \\
\hline 37.5 & 63.5 & 17.7 & 56.8 & 43.2 & 8.4 \\
\hline 25.0 & 75.0 & 7.6 & 54.8 & 45.2 & 9.2 \\
\hline
\end{tabular}

a Solvent, benzene $1 \mathrm{ml}$; total monomer amount $\left(\mathrm{M}_{1}+\mathrm{M}_{2}\right), 2 \mathrm{mmol}$; AIBN, $4 \times 10^{-2} \mathrm{mmol}$; temp, $60^{\circ} \mathrm{C}$; time, $8 \mathrm{~h}$.

the intercepts of the lines are positive seem to indicate the formation of contact chargetransfer complexes.

\section{Copolymerization of Glucal Derivatives with MAnh}

The copolymerization of glucals with MAnh was carried out at a monomer feed ratio of $1: 1$. DHP was also copolymerized with MAnh for comparison. The results are summarized in Table II.

All the glucals (TAG, TBG, and TMG) and DHP copolymerized with MAnh into powdery polymers which were soluble in dimethylformamide, acetone, tetrahydrofuran, and chloroform, and insoluble in hexane and water. The copolymers derived from TBG and TMG with MAnh were soluble whereas that from DHP-MAnh was insoluble in benzene. The copolymer of TAG-MAnh was not soluble but swelled in benzene, although the copolymerization of TAG and MAnh proceeded homogeneously in the solvent.

In all the IR spectra of these copolymers, strong absorptions of both acid anhydride at 1780 and $1860 \mathrm{~cm}^{-1}$ and cyclic ether at 1000 $1100 \mathrm{~cm}^{-1}$ were observed, indicating the glucals and DHP to have copolymerized with MAnh under these conditions.

As shown in Table II, the polymerizability of these cyclic ether monomers seemed to be relatively low compared with that of noncyclic vinyl ethers, probably owing to steric hin-

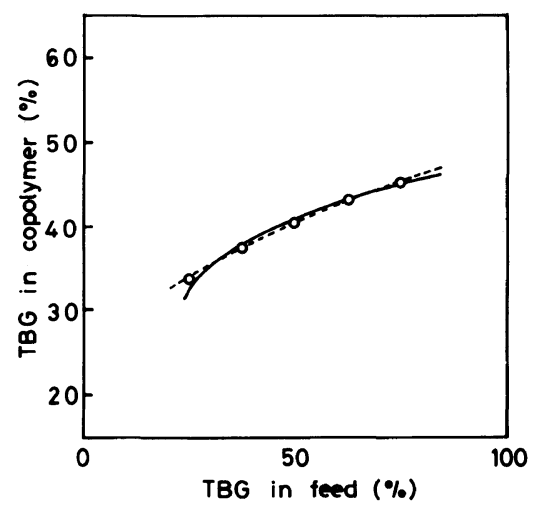

Figure 2. Composition diagrams for the copolymerization of tri- $O$-benzyl-D-glucal with maleic anhydride. (O), experimental results; (-), complex model analysis with $k_{1 \mathrm{C}} K / k_{11}=2.3$ and $k_{12} / k_{11}=0.7$; and $(----)$, penultimate model analysis with $r_{1}=0.1$ and $r_{1}{ }^{\prime}=0.8$.

drance at the propagating transition step and likely to the degradative chain transfer reaction involving the abstraction of allylic and/or benzylic hydrogens. TBG and DHP showed higher reactivity than TAG and TMG, although TBG has many active hydrogens. These results show that the copolymerization reactivity of these cyclic vinyl ethers are associated with the $K$ value, a measure of the complex formation.

\section{Analysis of Copolymerization Mechanism}

[A] By Copolymer Composition. TBG was chosen as a representative of the glucals and copolymerized with MAnh at a wide range of feed compositions. The copolymer compo- 
sition was determined by comparing the peak areas of phenyl protons of the polymer with that of dimethyl phthalate added as the internal reference in the ${ }^{1} \mathrm{H}$ NMR spectra. The results are shown in Table III and Figure 2.

The yield of the copolymer increased with the MAnh content in the monomer feed, while the number-average degree of polymerization was almost constant in a range 8.4-9.7 regardless of the monomer feed composition. The content of TBG in the copolymer increased with that in the monomer feed. In these systems, a tendency to form a $1: 1$ copolymer was not evident as in the case of the copolymerization of a noncyclic vinyl ether and MAnh, and the copolymers were always rich in MAnh units even at a monomer feed ratio of TBG:MAnh $=3: 1$. This polymerization behavior deviates from the concept of the terminal mechanism, but can be satisfactorily interpreted by the two different models as follows.

(1) Complex Model Analysis. The complex model which considers the participation of electron donor-acceptor charge-transfer complexes in the propagation is often applied to the systems giving alternating copolymers. Different propagation steps in this model can be presented as

$$
\begin{aligned}
& \sim \mathrm{M} \cdot+\mathrm{M} \underset{k_{11}}{\stackrel{k_{1 \mathrm{C}}}{\longrightarrow}} \sim \mathrm{M} . \\
& \sim \mathrm{M} \cdot+\mathrm{M} . \\
& \sim \mathrm{M} \cdot+\mathrm{T} \underset{k_{12}}{\longrightarrow} \sim \mathrm{T} . \\
& \sim \mathrm{T} \cdot+\mathrm{M} \underset{k_{21}}{\longrightarrow} \sim \mathrm{M} . \\
& \sim \mathrm{T} \cdot+\mathrm{C} \stackrel{k_{2 \mathrm{C}}}{\longrightarrow} \sim \mathrm{T} . \\
& \sim \mathrm{T} \cdot+\mathrm{T} \stackrel{k_{22}}{\longrightarrow} \sim \mathrm{T} .
\end{aligned}
$$

where $\mathrm{M}, \mathrm{T}$, and $\mathrm{C}$ represent $\mathrm{MAnh}, \mathrm{TBG}$, and the TBG-MAnh charge-transfer complex, respectively. In the TBG-MAnh copolymerization, it seems reasonable to assume $k_{22}=0$ because of the very low radical homopolymerizability of TBG. Then

$$
\frac{\mathrm{d}[\mathrm{M}]}{\mathrm{d}[\mathrm{T}]}=\frac{k_{11}[\mathrm{M} \cdot][\mathrm{M}]+k_{1 \mathrm{C}}[\mathrm{M} \cdot][\mathrm{C}]+k_{21}[\mathrm{~T} \cdot][\mathrm{M}]+k_{2 \mathrm{C}}[\mathrm{T} \cdot][\mathrm{C}]}{k_{1 \mathrm{C}}[\mathrm{M} \cdot][\mathrm{C}]+k_{12}[\mathrm{M} \cdot][\mathrm{T}]+k_{2 \mathrm{C}}[\mathrm{T} \cdot][\mathrm{C}]}
$$

The stationary-state assumption for different types of radicals gives the following equation.

$$
k_{12}[\mathrm{M} \cdot][\mathrm{T}]=k_{21}[\mathrm{~T} \cdot][\mathrm{M}]
$$

Then eq 1 can be written as

$$
\begin{aligned}
& \frac{\mathrm{d}[\mathrm{M}]}{\mathrm{d}[\mathrm{T}]} \\
& =1+\frac{k_{11}[\mathrm{M}]}{k_{1 \mathrm{C}} K[\mathrm{~T}][\mathrm{M}]+\frac{k_{2 \mathrm{c}} k_{12}}{k_{21}} K[\mathrm{~T}]^{2}+k_{12}[\mathrm{~T}]}
\end{aligned}
$$

Since the concentration of the TBG-MAnh complex is low and moreover, the TBG radical is supposed to have low stability, and hence low selectivity, the value of $\left(k_{2 \mathrm{C}} k_{12} / k_{21}\right) K[\mathrm{~T}]^{2}$ is reasonably assumed to be much smaller than $k_{1 \mathrm{C}} K[\mathrm{~T}][\mathrm{M}]+k_{12}[\mathrm{~T}]$. Then eq 3 may be simplified to

$$
\frac{\mathrm{d}[\mathrm{M}]}{\mathrm{d}[\mathrm{T}]}=1+\frac{[\mathrm{M}]}{\frac{k_{1 \mathrm{C}}}{k_{11}} K[\mathrm{~T}][\mathrm{M}]+\frac{k_{12}}{k_{11}}[\mathrm{~T}]}
$$

Various values were put in the places of $k_{1 \mathrm{C}} K / k_{11}$ and $k_{12} / k_{11}$ to obtain $\Delta[\mathrm{M}] / \Delta[\mathrm{T}]$ values by numeral integration that fit the experimental results; best values were found out as follows.

$$
\frac{k_{1 \mathrm{C}} K}{k_{11}}=2.3\left(1 \mathrm{~mol}^{-1}\right), \frac{k_{12}}{k_{11}}=0.70
$$

As shown in Figure 2, a plot according to eq 4 well agrees with the composition data.

(2) Penultimate Model Analysis. Taking into account the penultimate group effects, the chain propagating steps can be written as

$$
\begin{aligned}
& \sim \mathrm{MM} \cdot+\mathrm{M} \stackrel{k_{111}}{\longrightarrow} \sim \mathrm{MM} . \\
& \sim \mathrm{MM} \cdot+\mathrm{T} \stackrel{k_{112}}{\longrightarrow} \sim \mathrm{MT} . \\
& \sim \mathrm{MT} \cdot+\mathrm{M} \stackrel{k_{121}}{\longrightarrow} \sim \mathrm{TM} .
\end{aligned}
$$




$$
\begin{aligned}
& \sim \mathrm{MT} \cdot+\mathrm{T} \underset{k_{122}}{\longrightarrow} \sim \mathrm{TT} . \\
& \sim \mathrm{TM} \cdot+\mathrm{M} \stackrel{k_{211}}{\longrightarrow} \sim \mathrm{MM} . \\
& \sim \mathrm{TM} \cdot+\mathrm{T} \stackrel{k_{212}}{\longrightarrow} \sim \mathrm{MT} . \\
& \sim \mathrm{TT} \cdot+\mathrm{M} \stackrel{k_{221}}{\longrightarrow} \sim \mathrm{TM} . \\
& \sim \mathrm{TT} \cdot+\mathrm{T} \stackrel{k_{222}}{\longrightarrow \mathrm{TT} .}
\end{aligned}
$$

Assuming the stationary-state and $k_{122}=$ $k_{222}=0$, the following relationship is obtained.

$$
\frac{\mathrm{d}[\mathrm{M}]}{\mathrm{d}[\mathrm{T}]}=1+\frac{r_{1}{ }^{\prime} x\left(1+r_{1} x\right)}{r_{1}{ }^{\prime} x+1}
$$

where $\quad x=\frac{[\mathrm{M}]}{[\mathrm{T}]}, \quad r_{1}=\frac{k_{111}}{k_{112}}$, and $r_{1}^{\prime}=\frac{k_{211}}{k_{212}}$. Integration of eq 5 yields

$$
P \ln \left(\frac{x_{0}-m}{x-m}\right)+Q \ln \left(\frac{x_{0}-n}{x-n}\right)=\ln \frac{[\mathrm{T}]_{0}}{[\mathrm{~T}]}
$$

where

$$
\begin{gathered}
m=\frac{-b+\sqrt{b^{2}-4 a}}{2 a}, \quad n=\frac{-b-\sqrt{b^{2}-4 a}}{2 a} \\
a=r_{1} r_{1}{ }^{\prime}-r_{1}{ }^{\prime}, \quad b=2 r_{1}{ }^{\prime}-1, \\
\text { and } \quad Q=\frac{\frac{m}{r_{1}-1}+\frac{1}{r_{1}{ }^{\prime}\left(r_{1}-1\right)}}{m-n}, \\
\\
\qquad Q=\frac{-1}{r_{1}{ }^{\prime}\left(r_{1}-1\right)}-\frac{n}{r_{1}-1}
\end{gathered}
$$



Figure 3. Determination of the $r_{1}$ and $r_{1}{ }^{\prime}$ values of eq 6 for the copolymerization of tri- $O$-benzyl-D-glucal with maleic anhydride.
Plots of $r_{1}^{\prime} v s . r_{1}$ using eq 6 suggest the $r_{1}$ and $r_{1}^{\prime}$ values to be around 0.1 and 0.8 , respectively, as shown in Figure 3. The dotted line in Figure 2 was obtained according to eq 6 assuming $r_{1}=0.1$ and $r_{1}^{\prime}=0.8$. It nicely fits the experimental data as well as the plot by eq 4 .

As evident in Figure 2, the copolymerization of TBG with MAnh can be interpreted by both the conventional complex and penultimate models, despite the low degrees of polymerization, as far as the polymerization was analyzed on the basis of the copolymer composition.

[B] By Copolymerization Rate. According to the complex model, the decrease rate of the TBG concentraion in the copolymerization of TBG-MAnh can be presented as

$$
-\frac{\mathrm{d}[\mathrm{T}]}{\mathrm{d} t}=k_{1 \mathrm{C}} K[\mathrm{M} \cdot][\mathrm{T}][\mathrm{M}]+k_{12}[\mathrm{M} \cdot][\mathrm{T}]
$$

This shows the decrease rate of $[\mathrm{T}]$ to be proportional to $2.3 \times[\mathrm{T}][\mathrm{M}]+0.7 \times[\mathrm{T}]$, since $k_{1 \mathrm{C}} K / k_{11}=2.3$ and $k_{12} / k_{11}=0.70$. The rate is also expressed by the penultimate model as

$$
-\frac{\mathrm{d}[\mathrm{T}]}{\mathrm{d} t}=k_{112}[\mathrm{MM} \cdot][\mathrm{T}]+k_{212}[\mathrm{TM} \cdot][\mathrm{T}]
$$

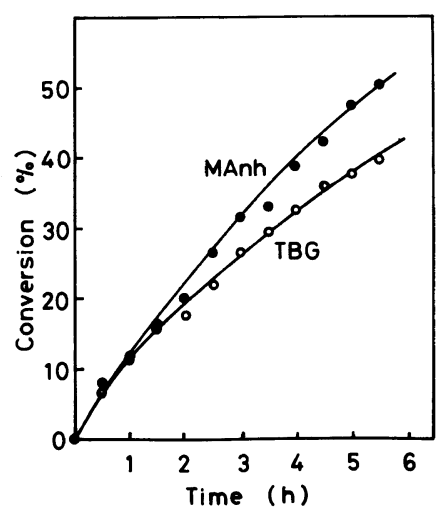

Figure 4. Monomer consumption curves in the copolymerization of tri- $O$-benzyl-D-glucal (TBG, $0.75 \mathrm{mmol}$ ) and maleic anhydride (MAnh, $1.25 \mathrm{mmol}$ ) at $60^{\circ} \mathrm{C}$ in benzene- $d_{6}(1 \mathrm{ml})$. 


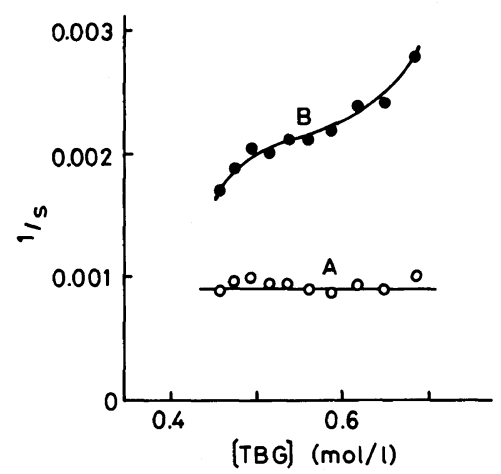

Figure 5. Dependences of $A$ and $B$ on the monomer concentration.

$$
\begin{gathered}
A=\frac{\frac{-\mathrm{d}[\mathrm{T}] / \mathrm{d} t}{\sqrt{[\mathrm{AIBN}] /[\mathrm{AIBN}]_{0}}}}{2.3[\mathrm{~T}][\mathrm{M}]+0.7[\mathrm{~T}]} \\
B=\frac{\frac{-\mathrm{d}[\mathrm{T}] / \mathrm{d} t}{\sqrt{[\mathrm{AIBN}] /[\mathrm{AIBN}]_{0}}}}{[\mathrm{~T}]}
\end{gathered}
$$

This indicates that the decrease in $[\mathrm{T}]$ is of the first order with respect to $T$ unless the $[\mathrm{T}] /[\mathrm{M}]$ value changes widely, and the relation was determined by the ${ }^{1} \mathrm{H}$ NMR spectra using $p$ dimethoxybenzene as the internal reference. The results are illustrated in Figure 4. Both monomers were smoothly consumed, and the rate of the copolymerization decreased with time. The ratio of $[\mathrm{T}]$ to $[\mathrm{M}]$ was almost constant at $0.60-0.70$ during the copolymerization. Assuming the total concentration of the macroradicals to be proportional to $[\mathrm{AIBN}]^{0.5}$, eq 7 can be written as,

$$
\frac{-\mathrm{d}[\mathrm{T}] / \mathrm{d} t}{\frac{\sqrt{[\mathrm{AIBN}] /[\mathrm{AIBN}]_{0}}}{2.3[\mathrm{~T}][\mathrm{M}]+0.7[\mathrm{~T}]}}=\text { constant } \quad(=A)
$$

Similarly, eq 8 can be transformed to

$$
\frac{\frac{-\mathrm{d}[\mathrm{T}] / \mathrm{d} t}{\sqrt{[\mathrm{AIBN}] /[\mathrm{AIBN}]_{0}}}}{[\mathrm{~T}]}=\text { constant } \quad(=B)
$$

$A$ and $B$ were calculated using $k_{\mathrm{d}}=$ $8.45 \times 10^{-6} \mathrm{~s}^{-1}$ for AIBN in benzene at $60^{\circ} \mathrm{C}$, and were plotted against [T]. As shown in Figure 5 , the value of $B$ clearly increased with the TBG concentration, indicating the decrease of $[\mathrm{T}]$ to be of an order higher than the first with respect to $T$, while the $A$ values were almost constant within experimental errors. Consequently, the complex model was found to fit the copolymerization of TBGMAnh much better than the penultimate model.

\section{REFERENCES}

1. E. Fischer and K. Zach, Sitz. Ber. Kgl. Preuss. Akad. Wiss., 16, 311 (1913).

2. K. Kurita, N. Hirakawa, and Y. Iwakura, Makromol. Chem., 180, 2331 (1979).

3. K. Kurita, K. Miyajima, T. Sannan, and Y. Iwakura, J. Polym. Sci., Polym. Chem. Ed., 18, 359 (1980).

4. K. Kurita, K. Murakami, K. Kobayashi, M. Takahashi, and Y. Koyama, Makromol. Chem., 187, 1359 (1986).

5. K. Kurita, N. Hirakawa, and Y. Iwakura, J. Polym. Sci., Polym. Chem. Ed., 18, 365 (1980).

6. K. Kurita, N. Hirakawa, H. Morinaga, and Y. Iwakura, Makromol. Chem., 180, 2769 (1979).

7. K. Kurita, N. Hirakawa, and Y. Iwakura, Makromol. Chem., 181, 1861 (1980).

8. S. Iwatsuki and Y. Yamashita, Makromol. Chem., 89, 205 (1965).

9. R. D. Kimbrough, Jr., W. P. Dickson, and J. M. Wilkinson, III, J. Polym. Sci., B, 2, 85 (1964).

10. Y. Inaki, S. Nozakura, and S. Murahashi, Kogyo Kagaku Zasshi, 26, 471 (1969).

11. B. Kamo, S. Kurashige, and S. Furukawa, Kogyo Kagaku Zasshi, 73, 580 (1970).

12. P. Boullanger, J. C. Martin, and G. Descotes, J. Heterocycl. Chem., 12, 91 (1975).

13. S. J. Eitelman, R. H. Hall, and A. Jordaan, J. Chem. Soc., 595 (1978).

14. R. Foster and C. A. Fyfe, Trans. Faraday Soc., 61, 1626 (1965). 